\title{
Evaluation of Urine Collection Methods for the Diagnosis of Urinary Tract Infection in Children
}

\author{
Candemir Karacan Nilgun Erkek Saliha Senel Suzan Akin Gunduz Gonul Catli \\ Betul Tavil \\ Department of Pediatrics, Dr. Sami Ulus Children's Hospital, Ankara, Turkey
}

\section{Key Words}

Urinary tract infection - Urine bag $\cdot$ Clean catch $\cdot$ Urethral

catheterization $\cdot$ Suprapubic aspiration

\begin{abstract}
Objective: To evaluate the accuracy of urine sample collection methods among children suspected of having urinary tract infections. Subjects and Methods: Four methods for urine sample collection were evaluated in 1,067 children aged $0-16$ years with suspected urinary tract infections over 2 months at Dr. Sami Ulus Children's Hospital. Within 30 min of collection, all specimens were sent to the laboratory, refrigerated and processed according to standard hospital microbiological procedures. Urine samples were analyzed using routine culture techniques. Results: At initial sending of the urine culture, 617 (57.8\%) had negative culture results, 145 (13.6\%) had positive culture results, and 305 (28.6\%) had evidence of bacterial contamination. Clean catch specimens showed a contamination rate of $14.3 \%$ and urethral catheterization specimens showed a similar contamination rate (14.3\%). However, urethral catheterization was preferred in only a small number of cases $(n=7)$. Suprapubic aspiration was also used in a small number of cases (n: 11) and the contamination rate for suprapubic aspiration was $9.1 \%$ (n: $1 / 11$ ). The contamination rate for sterile urine bag was $43.9 \%$, significantly higher than the other methods $(p<0.001)$. Conclu-
\end{abstract}

sion: Suprapubic aspiration showed the lowest contamination rate and sterile urine bag showed the highest contamination rate among 4 methods of urine sample collection. Contaminated specimens, needed to be repeated and this procedure increased the cost of urine culture. In conclusion, measures should be taken to reduce the contamination rate in our center. This is an area where further investigation is required.

Copyright $\odot 2010$ S. Karger AG, Basel

\section{Introduction}

Urinary tract infection (UTI) is one of the most common bacterial infections during childhood. UTI occurs in $5 \%$ of febrile infants without source of any infection [1]. UTIs are an important cause of chronic morbidity in children. Long-term complications include hypertension and reduced renal function secondary to renal scarring. Early treatment of UTI is very important to prevent these complications $[2,3]$. Additionally, the accurate diagnosis of UTI is necessary to ensure appropriate therapy for infected children and to avoid unnecessary therapy and prevent hospital admission for noninfected children [4].

A urine culture result is considered the gold standard for diagnosis of UTI, but collecting uncontaminated urine samples from young children is very difficult. Di-

\section{KARGER \\ Fax +4161306 1234 \\ E-Mail karger@karger.ch}

www.karger.com
(C) 2010 S. Karger AG, Basel

1011-7571/10/0193-0188\$26.00/0

Accessible online at:

www.karger.com/mpp
Saliha Senel

Dr. Sami Ulus Children's Hospital

Telsizler, Diskapi

TR-06080 Ankara (Turkey)

Tel. +90 312305 6014, Fax +90 312317 0353, E-Mail drsaliha007@yahoo.com.tr 
agnosis of UTI requires collection of urine generally by 1 of 4 methods: sterile urine bag, urethral catheterization $(\mathrm{CATH})$, suprapubic aspiration (SPA), or clean catch (CC). Both CATH and SPA are thought to be the most reliable methods by minimizing false-positive results, but these methods are more invasive and painful than the others. The sterile urine bag is a noninvasive and easy alternative, although this method results in a high contamination rate [4-6].

Choosing the appropriate urine collection method provides a difficult decision and little is known about how this decision is made. In this study, urine sample collection methods were evaluated in 1,067 children who were admitted to a busy children's hospital. Our objective was to answer the following questions in this study: (1) Which urine collection techniques do pediatricians use to diagnose UTIs in children between the ages of $0-16$ years? (2) What is the contamination rate with each of the urine collection techniques? (3) Which urine collection techniques are preferred in different age groups? (4) How many times did urine collection need to be repeated to provide a definitive result?

\section{Materials and Methods}

The study was performed over 2 months at the Dr. Sami Ulus Children's Hospital. Children aged $0-16$ years with suspected urinary tract infection were enrolled in this study. In 1,067 children, urine specimens for culture were obtained by sterile urine bag, midstream CC, CATH or SPA.

Initially, urine bags were placed by trained nurses, using standard perineal cleansing procedures, but if urine bags were separated from the skin or there were problems of leakage or stool contamination, parents were taught to place them according to cleansing procedures. CC specimens were also obtained by parents. For CC specimens, parents had a sterile urine container ready to collect urine, taking care that the perineum did not touch the inside of the container. Urine samples were obtained sterilely in SPA and CATH by specialized personnel using an aseptic technique.

Within 30 min of collection, all specimens were sent to the laboratory, processed on blood agar and eosin methylene blue (EMB) medium by standard loop method and incubated at $37^{\circ} \mathrm{C}$ overnight. Urine samples were analyzed using the criteria established by the Clinical Laboratory Standards Institute (CLSI; formerly National Committee for Clinical Laboratory Standards [NCCLS]) protocol [7]. A positive urine culture was defined as the growth of a single pathogen of more than $10^{5}$-colony forming units $(\mathrm{CFU}) / \mathrm{ml}$ in a sterile urine bag and CC specimens, more than $10^{4}-\mathrm{CFU} / \mathrm{ml}$ in urine specimens obtained by CATH [8], and any number of colonies in a sample obtained by SPA [9]. Urine cultures which had been interpreted as 'contaminated' by the laboratory were considered to be contaminated.

Evaluation of Urine Collection Methods in Children
The data were analyzed using the Statistical Package of Social Sciences for Windows, version 10.0 (SPSS Inc., Chicago, Ill., USA) program. Pearson's $\chi^{2}$ and Fisher's exact tests were used to compare differences between groups. $\mathrm{p} \leq 0.05$ was considered significant.

\section{Results}

Among 1,067 children, 88 were younger than 1 month, 126 were between 1 and 6 months, 318 were between 7 and 24 months, and 535 were older than 24 months of age. There were 521 (48.9\%) girls. Of these 1,067 children, 951 (89.1\%) were admitted to the outpatient clinic and 116 (10.9\%) were admitted to the inpatient clinic. A sterile urine bag was used in 517 (48.5\%) children for urine collection, CC was used in 532 (49.9\%) children, SPA in 11 (1\%) children, and CATH in only $7(0.7 \%)$ children. After initial sending of the urine culture, 617 (57.8\%) had negative culture results, 145 (13.6\%) had positive culture results, and 305 (28.6\%) had evidence of bacterial contamination.

Among 88 newborns (children younger than 1 month of age), a sterile urine bag was used in 73 (83\%), SPA was used in $10(11.4 \%)$, CATH was used in $4(4.5 \%)$, and CC in only 1 (1.1\%). The most preferred method for urine collection was a sterile urine bag in the newborn period. SPA, a more invasive method than the others was used mostly in the newborn period ( $\mathrm{n}=10 / 11,90.9 \%$ ) in respect to other age groups. Among 126 children between the ages of 1 and 6 months, a sterile urine bag was used in $124(98.4 \%)$ children, SPA was used in $1(0.8 \%)$, CATH was used in $1(0.8 \%)$, and CC was not used in this age group. The most preferred method for urine sample collection was the sterile urine bag. SPA and CATH were not chosen practically for urine sample collection in this age group. Among 318 children between the ages of 7-24 months, the sterile urine bag was used in $279(87.7 \%)$ children, CC was used in 38 (11.9\%), CATH was used in 1 $(0.3 \%)$, and SPA was not used in this age group. The sterile urine bag was the most preferred method again for urine sample collection in children between 7 and 24 months. Among 535 children older than 24 months, CC was used in 493 (92.1\%), sterile urine bag was used in 41 (7.7\%) children, CATH was used in $1(0.2 \%)$, and SPA was not used in this age group. The most preferred method for urine sample collection was CC in children older than 24 months (table 1).

CC specimens showed a contamination rate of $14.3 \%$ $(\mathrm{n}=76 / 532)$ and CATH specimens showed a similar contamination rate $(14.3 \%, \mathrm{n}=1 / 7)$. However, CATH was 
Table 1. Contamination and repetition rates of urine collection methods

\begin{tabular}{lcl}
\hline Urine collection methods & $\begin{array}{l}\text { Contami- } \\
\text { nation } \\
\text { rate, } \%\end{array}$ & $\begin{array}{l}\text { Repetition rate } \\
\text { with contaminated } \\
\text { specimens, \% }\end{array}$ \\
\hline Sterile urine bag & $43.9^{*}$ & 44.9 \\
Clean catch & 14.3 & 50.0 \\
Suprapubic aspiration & 9.1 & - \\
Catheterization & 14.3 & - \\
\hline
\end{tabular}

${ }^{*} \mathrm{p}<0.001$.

preferred in only a small number of cases $(n=7)$. SPA was also used in a small number of cases $(\mathrm{n}=11)$ and the contamination rate for SPA was 9.1\% $(n=1 / 11)$. The contamination rate for sterile urine bags was $43.9 \%(n=227 / 517)$, significantly higher than the other methods $(\mathrm{p}<0.001)$. SPA showed the lowest contamination rate $(9.1 \%)$ (table 1). Children with contaminated specimens had not been started on prior antibiotics.

Of the 76 contaminated CC specimens, 38 (50\%) were repeated. All contaminated specimens needed to be repeated, but families repeated only half of the specimens. Thirty-six (94.7\%) repeated CC specimens were concluded at the 2 nd and $2(5.3 \%)$ were concluded at the 3rd sampling of the specimens. At the second repeat of these 36 CC specimens, $12(33.3 \%)$ were positive and the others were negative. At the third repeat of $2 \mathrm{CC}$ specimens, all were positive. Of the 227 contaminated sterile urine bag specimens, 102 (44.9\%) were repeated. Ninety-four (92.2\%) repeated sterile urine bag specimens were concluded at the 2 nd and 8 (7.8\%) were concluded at the 3rd sampling of the specimens. CATH $(n=6)$ and SPA $(n=$ 5) were also selected methods for repeat sample collection in cases of contamination. At the second repeat of these 94 sterile urine bag specimens, 28 (29.8\%) were positive and the others were negative. At the third repeat of 8 sterile urine bag specimens, all were positive for UTI.

\section{Discussion}

The sterile urine bag is a common sampling method especially for babies and infants. It has several advantages over other methods but high risk of contamination constitutes a disadvantage of this method [4]. CC is a noninvasive and easy method, but it is very difficult to carry out in younger children. It is generally reserved for older children who are able to co-operate [10]. CC has a greater risk that the mother or child will rub the container on the perineal skin or handle the inside of the container [11]. CATH is performed by insertion of a catheter through the urethra into the bladder. CATH is considered more accurate but technically invasive and painful, which is a source of parental anxiety. In addition, CATH may risk introducing bacteria into the urinary tract and has been shown to be a risk factor for septicemia in neonates [12]. Compared with CATH, bag urine cultures were 3 times more likely to cause diagnostic uncertainty because of ambiguous cultures [12]. SPA has been regarded as the gold standard urine collection method. It is performed with a needle attached to a syringe inserted through the lower abdomen into the bladder with ultrasonography guidance. While the probability of a true infection with a positive culture obtained with SPA is approximately $99 \%$, this method is technically the most challenging and is associated with the lowest rate of success [10]. The success rate of obtaining urine by SPA can be low $[10,13]$. In our study, the most preferred method for urine sample collection was the sterile urine bag in the newborn period and also in the infancy period. The contamination rate for sterile urine bag was $43.9 \%$ in our study, which means it was 3 times more likely to result in a contaminated culture, which is consistent with reports in the literature [12]. CC was the most preferred method in children older than 24 months and CC specimens showed a contamination rate of $14.3 \%$, which is in accordance with the literature [11]. SPA was mostly used in the newborn period with respect to other age groups.

Contaminated specimens can hide true infections [14] and we found that $30 \%$ of contaminated specimens were true infections in our study, as is reported in the literature. Contaminated specimens need to be repeated and this procedure increases the cost of urine culture [4]. Unnecessary investigations may lead to unnecessary antibiotics and hospitalization $[4,15]$. Children with contaminated specimens had not been started on prior antibiotics in our center. We repeated urine cultures till we obtained pure urine cultures.

In our study, the contamination rate for sterile urine bag was significantly higher than the other methods, in accordance with the literature. CC specimens and CATH specimens showed similar contamination rates. They failed to show significant differences in the contamination rates perhaps because CATH was preferred in only a small number of cases. SPA, also used in a small number of cases, showed the lowest contamination rate. Our hos- 
pital is a busy center with nearly 360,000 outpatient and emergency department visits annually and we worked with half of the required number of nurses because of our Health Ministry facilities. As a result, parents took part in urine collection. Cleansing materials with antimicrobial properties interfered with the bag urine culture, and false-negative cultures may have resulted. Urine specimens taken by educated health staff instead of parents, with a suitable cleansing agent which does not affect bacteria present in the urine, could reduce the false urine sampling and contamination rates in our center. Disposable nappies and urine collection pads as alternative urine collection methods may also be tried in our center $[15$, $16]$.

The use of CATH and SPA may be increased especially during the infancy period. On the other hand, parental preference is an important factor in choosing the urine collection method in our center. CATH and SPA are invasive techniques, so to insist on these procedures has the potential to lead to parental anxiety and rejection by our population. Urine bag screening may be chosen in lowrisk patients to reduce the number of CATHs, but in highrisk patients (e.g. recurrent UTI, reflux, impaired renal function) a catheter specimen should be sent for culture [13]. A limitation of our study was that it was conducted at a single center.

\section{Conclusion}

There was a high contamination rate with urine bag culture. Measures should be taken to reduce the contamination rates in our center. Errors can be minimized if bag urine cultures are performed appropriately by skilled professionals. This is an area where further investigation is required. Further research should also be undertaken to survey provider beliefs and barriers to the use of other urine collection methods.

\section{References}

1 Hoberman A, Chao HP, Keller DM, Hickey $\mathrm{R}$, Davis HW, Ellis D: Prevalence of urinary tract infection in febrile infants. J Pediatr 1993; 123:17-23.

-2 Downs SM: Technical report: urinary tract infections in febrile infants and young children. The Urinary Tract Subcommittee of the American Academy of Pediatrics Committee on Quality Improvement. Pediatrics 1999; 103:e54.

- 3 Al-Eisa A, Al-Mosawi M, Gupta R, D’Souza TM, Sharma PN: Attitudes of general practitioners and pediatricians to management of acute pyelonephritis in children. Med Principles Pract 2000;9:119-124.

4 Al-Orifi F, McGillivray D, Tange S, Kramer MS: Urine culture from bag specimens in young children: are the risks too high? J Pediatr 2000;137:221-226.

5 Grisaru-Soen G, Goldman R, Barzilai A, Lo$\tan \mathrm{D}$, Keller N: False-positive urine cultures using bag collection. Clin Pediatr (Philadelphia) 2000;39:499-500.
-6 Li PS, Ma LC, Wong SN: Is bag urine culture useful in monitoring urinary tract infection in infants? J Paediatr Child Health 2002;38: 377-381.

7 National Committee for Clinical Laboratory Standards: Performance standards for antimicrobial susceptibility testing. Twelfth informational supplement. NCCLS document M100-S12. Wayne, National Committee for Clinical Laboratory Standards, 2002.

$\checkmark 8$ Guidoni EB, Berezin EN, Nigro S, Santiago NA, Benini V, Toporovski J: Antimicrobial agent resistance patterns of pediatric community-acquired urinary infections. Braz J Infect Dis 2008;12:321-323.

$\checkmark 9$ Goldraich NP, Manfroi A: Febrile urinary tract infection: Escherichia coli susceptibility to oral antimicrobials. Pediatr Nephrol 2002; 17:173-176.

10 Chang SL, Shortliffe LD: Pediatric urinary tract infections. Pediatr Clin North Am 2006;53:379-400.

11 Alam MT, Coulter JB, Pacheco J, Correia JB, Ribeiro MG, Coelho MF, Bunn JE: Comparison of urine contamination rates using three different methods of collection: clean-catch, cotton wool pad and urine bag. Ann Trop Paediatr 2005;25:29-34.
-12 Schroeder AR, Newman TB, Wasserman RC, Finch SA, Pantell RH: Choice of urine collection methods for the diagnosis of urinary tract infection in young, febrile infants. Arch Pediatr Adolesc Med 2005;159:915922

13 McGillivray D, Mok E, Mulrooney E, Kramer MS: A head-to-head comparison: 'cleanvoid' bag versus catheter urinalysis in the diagnosis of urinary tract infection in young children. J Pediatr 2005; 147:451-456.

14 Macfarlane PI, Houghton C, Hughes C: Pad urine collection for early childhood urinarytract infection. Lancet 1999;354:571.

15 Loane V: Obtaining urine for culture from non-potty-trained children. Paediatr Nurs 2005; 17:39-42.

16 Rao S, Houghton C, Macfarlane PI: A new urine collection method; pad and moisture sensitive alarm. Arch Dis Child 2003;88: 836 